\title{
Crescimento e composição química de dez espécies de microalgas marinhas em cultivos estanques
}

\author{
Growth and chemical composition of ten species of marine microalgae in batch cultures
}

\author{
Viviane Borges Campos ${ }^{\mathrm{I} *}$ Elisabete Barbarino ${ }^{\mathrm{I}}$ Sergio de Oliveira Lourenço ${ }^{\mathrm{I}}$
}

\section{RESUMO}

\begin{abstract}
Microalgas apresentam diversas aplicações econômicas consagradas, como usos na aquicultura e na indústria de alimentos, havendo buscas por novos usos, como a geração de biomassa para produção de biodiesel. As possíveis aplicações estão diretamente relacionadas à taxa de crescimento e ao perfil químico das espécies. Assim, a seleção de condições que promovam o aproveitamento da biomassa algácea é fundamental para sua utilização econômica. Neste estudo, 10 espécies de microalgas marinhas foram cultivadas e comparadas quanto ao crescimento e à composição química. Foram observadas diferenças na velocidade de crescimento, com espécies de células menores crescendo mais rapidamente que microalgas maiores. Teores de proteínas, carboidratos, lipídeos e pigmentos fotossintetizantes variaram amplamente entre as espécies, sendo as proteínas as substâncias mais abundantes. Todas as espécies apresentaram concentrações de ácidos aminados semelhantes, sendo os ácidos aspártico e glutâmico os mais abundantes. Algumas espécies apresentaram altas concentrações de ácidos graxos de importância econômica, como os ácidos eicosapentaenoico e linoleico. $O$ balanço dos resultados indica que há poucas tendências gerais relacionadas a grandes grupos taxonômicos.
\end{abstract}

Palavras-chave: algas microscópicas, aplicações, biomassa, cultura, perfil químico.

\section{ABSTRACT}

Microalgae show several economic applications, such as uses in aquaculture and in food industry, and there is a search for new uses, such as the biomass production to convert into biodiesel. All possible applications are directly linked to growth rate and the chemical profile of the species. Thus, the selection of conditions to promote a better use of algal biomass is fundamental for economic purposes. In this study, 10 species of marine microalgae were cultured and compared for growth and chemical composition. Remarkable differences of growth performance have been observed, with species with small cell volumes growing faster than species with large cell volumes. Levels of protein, carbohydrate, lipid and photosynthetic pigments varied widely, and proteins were identified as the most abundant substances. Some species showed high concentrations of fatty acids of economic importance, such as eicosapentaenoic and linoleic acids. The concentrations of amino acids were similar among species. In all microalgae, glutamic and aspartic acids were the most abundant amino acids. An overall evaluation of the results indicates that few general trends related to the taxonomy of algal groups were recognized.

Key words: microalgae, culture, growth, chemical composition.

\section{INTRODUÇÃO}

Microalgas são seres fotossintetizantes ubíquos em sistemas aquáticos, envolvendo enorme diversidade de formas e funções ecológicas, sendo também aproveitadas em atividades econômicas. De forma geral, apresentam elevadas taxas de crescimento, condição que proporciona alta produção de biomassa em intervalos de tempo curtos. A produtividade de sistemas algáceos é superior a de quaisquer culturas agrícolas conhecidas (PULZ \& GROSS, 2004). A aplicação de microalgas em aquicultura constitui-se numa prática centenária (LOURENÇO, 2006), sendo necessárias direta ou indiretamente no cultivo de animais marinhos (DE PAWN et al., 1984). Além disso, desde a década de 1970, algumas espécies vêm sendo cultivadas para produzir encapsulados para alimentação humana (LOURENÇO, 2006). Diversas espécies são cultivadas para produzir substâncias específicas (ex.:

IDepartamento de Biologia Marinha, Laboratório de Fisiologia e Cultivo de Algas, Universidade Federal Fluminense (UFF), Campus Valonguinho, CP 100644, 24001-970, Niterói, RJ, Brasil. E-mail: borgescamposv@yahoo.com.br.*Autor para correspondência. 
ácidos graxos, ácidos aminados e pigmentos fotossintetizantes) e não para a utilização da biomassa integral.

Atualmente, muitos estudos estão sendo conduzidos sobre a utilização de microalgas para a produção de biocombustíveis associada à captura de $\mathrm{CO}_{2}$ (CHISTI, 2007) e ao tratamento de águas residuais (SKJANES et al., 2007), os quais são fundamentados na maior eficiência desses organismos para capturar energia solar e converter em biomassa, em comparação com as plantas (LI et al., 2008).

Muitas microalgas são potencialmente úteis para produção em grande escala; entretanto, a escolha de espécies envolve questões diversas, tais como a velocidade de crescimento e sua composição química. Essas variáveis são influenciadas por alguns fatores como o meio de cultura utilizado, a idade do cultivo, a intensidade luminosa, a temperatura, a salinidade e o fotoperíodo (BROWN et al., 1997).

Nesse sentido, existe uma demanda por pesquisas prospectivas de fisiologia e composição química de microalgas para identificar espécies úteis a aplicações comerciais. Neste estudo, 10 espécies de microalgas predominantemente nativas foram avaliadas quanto ao crescimento e à composição química.

\section{MATERIAL E MÉTODOS}

Foram estudadas 10 espécies de microalgas marinhas de diferentes grupos taxonômicos: as clorófitas Chlorella minutissima, Dunaliella tertiolecta e Tetraselmis gracilis; as diatomáceas Phaeodactylum tricornutum e Skeletonema costatum; a cianobactéria Synechococcus subsalsus; a criptofícea Rhodomonas sp.; a haptofícea Isochrysis galbana; a eustigmatofícea Nannochloropsis oculata e o dinoflagelado Prorocentrum minimum. Informações sobre grupos taxonômicos de algas podem ser obtidas em LOURENÇO (2006).

As espécies foram cultivadas em meio Conway (WALNE, 1966), de forma estanque, em quadruplicada, em balões volumétricos de 3,0 litros, contendo 2,4 litros de cultura, em irradiância de $300 \mu \mathrm{E}$ $\mathrm{m}^{-2} \mathrm{~s}^{-1}$, fotoperíodo de 12 horas, temperatura de $20 \pm 2^{\circ} \mathrm{C}$ e salinidade média de 33,2ups. Diariamente, no início do período de luz, alíquotas dos cultivos foram retiradas para realização de contagem celular.

As amostragens para medições de biovolumes celulares (HILLEBRAND et al., 1999) e análises químicas foram realizadas no início da fase estacionária de crescimento. Volumes de 700-850ml dos cultivos foram centrifugados, e os concentrados foram congelados $\left(-18^{\circ} \mathrm{C}\right)$, sendo posteriormente liofilizados para a realização de análises de proteínas, carboidratos, lipídeos/ácidos graxos e ácidos aminados. Para análises de pigmentos, $15-30 \mathrm{ml}$ dos cultivos foram filtrados em filtros de fibra de vidro. O meio de cultura filtrado foi destinado às determinações das concentrações dos sais nutrientes dissolvidos.

As proteínas hidrossolúveis foram quantificadas segundo LOWRY et al. (1951), usando albumina bovina como padrão. A extração dos carboidratos seguiu MYKLESTAD \& HAUG (1972), e a quantificação, DUBOIS et al. (1956), empregando-se glicose como padrão. Os pigmentos fotossintéticos foram extraídos em "acetona 90\%", com manutenção das amostras a $4^{\circ} \mathrm{C}$, por 20 horas, no escuro. A análise de clorofila $a$ se deu de acordo com LORENZEN (1967), e a de carotenoides totais, segundo STRICKLAND \& PARSONS (1968). A fração lipídica foi obtida de acordo com FOLCH et al. (1957) e determinada por gravimetria, após a completa evaporação do solvente.

Os ácidos graxos foram determinados segundo HARTMAN \& LAGO (1973) e processados em cromatógrafo em fase gasosa CG, modelo 500, dotado de detector e ionizador de chama, empregandose hidrogênio como gás de arraste. Os ácidos graxos foram identificados por comparação com padrões. As análises de ácidos aminados totais foram realizadas por cromatografia de troca iônica em analisador de ácidos aminados Beckman, modelo 7300, mediante prévia hidrólise das amostras com $\mathrm{HCl}$ a $6 \mathrm{~N}$, a $110^{\circ} \mathrm{C}$, por 22 horas, em ampolas fechadas a vácuo, com adição de norleucina como padrão interno. As condições analíticas permitiram a determinação de todos os ácidos aminados, exceto triptofano, e acarretou destruição parcial de cisteína + cistina e metionina. Após a hidrólise, as amostras foram secas e solubilizadas em solução tampão $\mathrm{Na}-\mathrm{S} \AA$, pH 2, 2, filtradas e injetadas no analisador de ácidos aminados, dotado de integrador/ registrador automático para obtenção dos aminogramas. Os ácidos aminados foram identificados por meio da comparação com padrões conhecidos das substâncias.

Os resultados foram submetidos à análise de variância (ANOVA), seguida de comparações múltiplas de Tukey (ZAR 1996) $(\mathrm{P} \leq 0,05)$, utilizando o programa Statistica for Windows. A homocedasticidade dos dados foi testada previamente, e transformações adequadas (logarítmicas) foram realizadas nas situações em que foram necessárias.

\section{RESULTADOS E DISCUSSÃO}

As curvas de crescimento e os dias de amostragem das microalgas estão representados na figura 1, e os volumes celulares e a composição química das espécies, na tabela 1 . O crescimento variou 


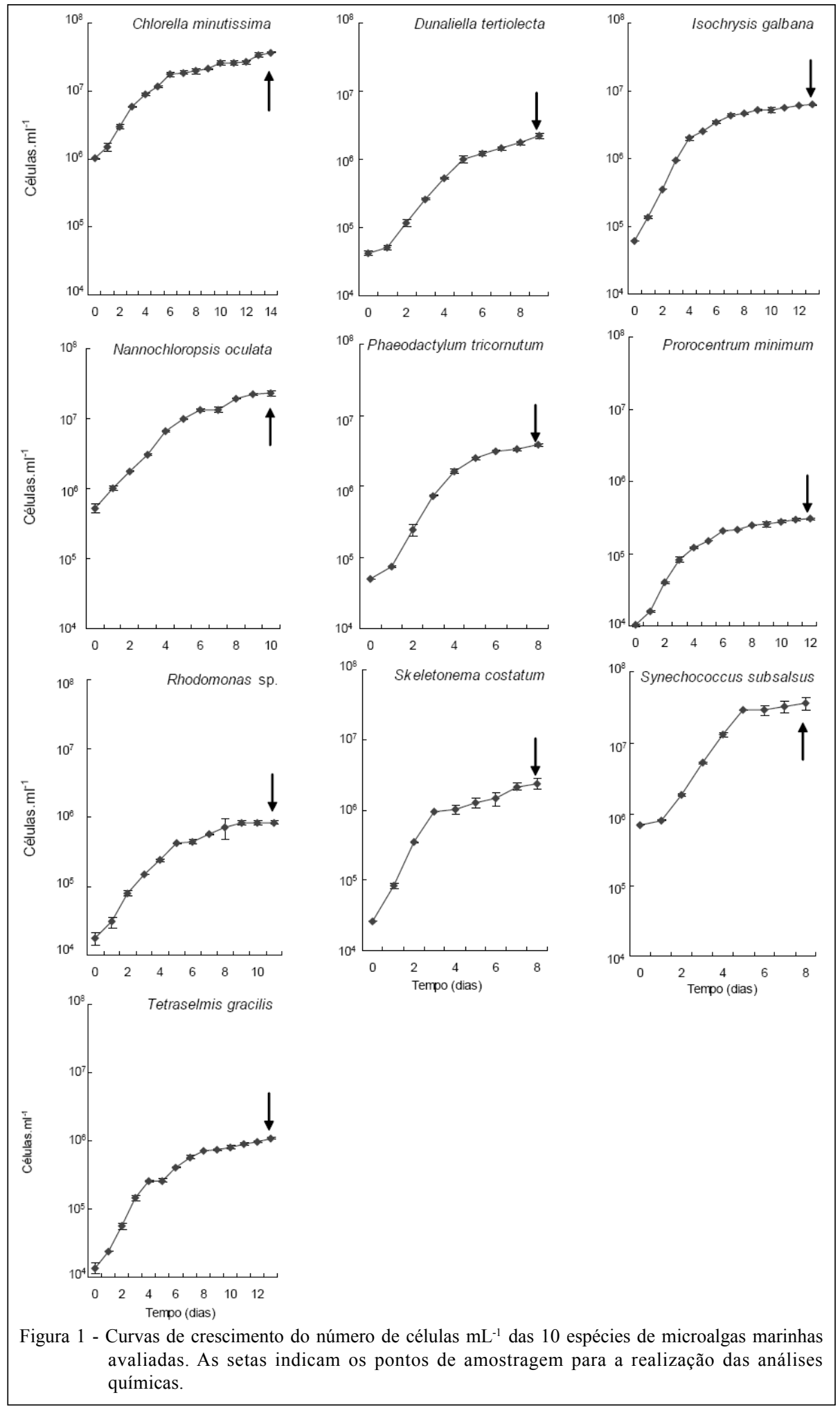

amplamente entre as microalgas. Espécies dotadas de menores biovolumes celulares (S. subsalsus e $\boldsymbol{C}$. minutissima) apresentaram maiores velocidades de crescimento, ao passo que $\boldsymbol{P}$. minimum, espécie de maior volume celular, apresentou baixa velocidade de crescimento. Tais resultados devem estar associados 
Tabela 1 - Composição de 10 espécies de microalgas cultivadas em meio Conway, na fase estacionária de crescimento ${ }^{1}$.

\begin{tabular}{|c|c|c|c|c|c|c|}
\hline Espécies & Volume celular & Proteínas & Carboidratos & Lipídeos & Clorofila $a$ & Carotenoides \\
\hline & $\left(\mu \mathrm{m}^{3}\right)$ & \multicolumn{5}{|c|}{ 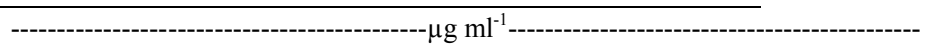 } \\
\hline Chlorella minutissima & $1,2 \pm 0,5$ & $14,9 \pm 1,3^{\mathrm{c}}$ & $6,6 \pm 0,3^{\mathrm{f}}$ & $2,2 \pm 0,2^{\mathbf{d}}$ & $0,13 \pm 0,02^{\mathbf{d}}$ & $0,07 \pm 0,01^{\mathrm{e}}$ \\
\hline Dunaliella tertiolecta & $127 \pm 37$ & $26,0 \pm 1,3^{\mathbf{b}}$ & $9,2 \pm 0,5^{\mathbf{e}}$ & $2,4 \pm 0,1^{\mathbf{d}}$ & $1,12 \pm 0,10^{\mathbf{a}}$ & $0,66 \pm 0,05^{\mathrm{a}}$ \\
\hline Isochrysis galbana & $58,3 \pm 13,5$ & $29,4 \pm 1,9^{\mathbf{a}}$ & $18,6 \pm 1,7^{\mathbf{b}}$ & $4,5 \pm 0,2^{\mathbf{a}}$ & $0,32 \pm 0,03^{c}$ & $0,10 \pm 0,02^{\mathrm{e}}$ \\
\hline Nannochloropsis oculata & $10,9 \pm 2,3$ & $21,3 \pm 1,3^{\mathbf{b}}$ & $11,0 \pm 0,3^{\mathbf{d}}$ & $3,4 \pm 0,4^{\mathbf{b}}$ & $0,90 \pm 0,02^{\mathbf{b}}$ & $0,30 \pm 0,04^{\mathrm{c}}$ \\
\hline Phaeodactylum tricornutum & $121 \pm 38,2$ & $23,3 \pm 0,8^{\mathbf{b}}$ & $13,1 \pm 0,5^{\mathrm{c}}$ & $4,0 \pm 0,3^{\mathbf{b}}$ & $1,03 \pm 0,03^{\mathrm{a}}$ & $0,44 \pm 0,01^{\mathbf{b}}$ \\
\hline Prorocentrum minimum & $1.411 \pm 324$ & $30,9 \pm 2,2^{\mathbf{a}}$ & $17,6 \pm 1,1^{\mathbf{b}}$ & $3,5 \pm 0,4^{\mathbf{b}}$ & $0,19 \pm 0,01^{\mathbf{d}}$ & $0,09 \pm 0,01^{\mathrm{e}}$ \\
\hline Rhodomonas sp. & $188 \pm 55,7$ & $20,1 \pm 0,9^{\mathbf{b}}$ & $5,8 \pm 0,1^{\mathrm{f}}$ & $2,2 \pm 0,1^{\mathbf{d}}$ & $0,37 \pm 0,01^{\mathrm{c}}$ & $0,34 \pm 0,02^{\mathrm{c}}$ \\
\hline Skeletonema costatum & $49,4 \pm 12,8$ & $14,9 \pm 0,8^{c}$ & $8,4 \pm 0,4^{\mathrm{e}}$ & $1,0 \pm 0,0^{\mathbf{e}}$ & $0,18 \pm 0,02^{\mathbf{d}}$ & $0,04 \pm 0,00^{f}$ \\
\hline Synechococcus subsalsus & $1,71 \pm 0,7$ & $21,2 \pm 0,8^{\mathbf{b}}$ & $12,9 \pm 0,4^{\mathrm{c}}$ & $3,0 \pm 0,0^{c}$ & $0,34 \pm 0,05^{\mathrm{c}}$ & $0,19 \pm 0,02^{d}$ \\
\hline Tetraselmis gracilis & $391 \pm 43,7$ & $33,6 \pm 2,5^{\mathrm{a}}$ & $23,1 \pm 1,4^{\mathbf{a}}$ & $5,3 \pm 0,6^{\mathbf{a}}$ & $0,93 \pm 0,09^{\mathbf{b}}$ & $0,56 \pm 0,05^{\mathbf{a}}$ \\
\hline
\end{tabular}

${ }^{1}$ Os dados referem-se à média de três ou quatro repetições \pm desvio padrão. Letras sobrescritas distintas indicam diferença significativa pelo teste de Tukey $(\mathrm{P}=0,05)$. Os dados de volume celular não foram analisados estatisticamente.

à maior eficiência na absorção de nutrientes por células menores, devido à maior razão superfície-volume destas (LAVÍN \& LOURENÇO, 2005), bem como a diferenças em atividade de enzimas associadas ao metabolismo de nutrientes (LOURENÇO, 2006).

As proteínas foram os componentes mais abundantes em todas as espécies, como observado por BECKER (2007) e BROWN et al. (1997). Concentrações de carboidratos e lipídeos foram mais baixas se comparadas com os dados da literatura sobre composição química de microalgas na fase estacionária de crescimento. Essa condição deve ser consequência da grande disponibilidade de nitrogênio no meio de cultura $(1.176 \mu \mathrm{M})$, parcialmente consumido ao final dos experimentos (dados não apresentados). $\mathrm{O}$ excesso de nitrogênio deve ter conduzido as espécies a apresentarem elevada produção de proteínas e menor síntese de carboidratos e lipídeos, interpretação respaldada por LOURENÇO et al. (2004). Dessa forma, esse delineamento experimental favoreceu o acúmulo de proteínas por essas espécies e pode servir como base para estudos posteriores voltados para o uso de microalgas como fonte de proteínas.

\section{T. gracilis, P. minimum e I. galbana} apresentaram maiores concentrações de proteínas (Tabela 1), concordando com RENAULD et al. (1999), que observaram altos valores proteicos em prasinofíceas e primnesiofíceas. Apesar da baixa taxa de crescimento, $\boldsymbol{P}$. minimum exibiu altos teores de proteínas, sugerindo alta eficiência na conversão de nutrientes em biomassa. As baixas concentrações de proteínas em C. minutissima devem resultar do menor crescimento dessa espécie quando cultivada com nitrato como fonte de nitrogênio (LOURENÇO et al., 2002). Por outro lado, o crescimento lento e as baixas taxas de proteínas e outras substâncias na diatomácea S. costatum parecem refletir carência de silício, exigido por essa espécie para compor suas frústulas em proporção N:Si de 1,0:0,5 a 1,0:1,0 (BRZEZINSKI, 1985). O meio Conway apresenta proporção N:Si de 1,0:0,25 e provavelmente deve ser enriquecido com mais silício para proporcionar um aumento mais acentuado da biomassa dessa diatomácea. Concentrações relativamente baixas de carboidratos e lipídeos registradas na maioria das espécies refletem o acoplamento entre o metabolismo de carbono e de nitrogênio, pois a acumulação de nitrogênio e proteínas naturalmente acarreta redução de substâncias de reserva, como demonstrado por TURPIN (1991). T. gracilis apresentou a maior concentração de carboidratos, concordando com BROWN et al. (1997), que observaram altas concentrações em prasinofíceas. Maiores rendimentos lipídicos foram observados em T. gracilis e I. galbana, refletindo a alta biomassa alcançada e acumulação de reservas lipídicas, respectivamente.

Concentrações de pigmentos fotossintetizantes refletem o ajuste cromático de espécies às condições de irradiância, sendo características de cada espécie (LOURENÇO, 2006). Maiores concentrações foram observadas em $\boldsymbol{P}$. tricornutum e D. tertiolecta. O mesmo foi observado por LOURENÇO et al. (2004), para D. tertiolecta cultivada em condições semelhantes.

A composição de ácidos graxos variou expressivamente (Tabelas 2 e 3), sendo os saturados (AGS) mais abundantes que os monoinsaturados (AGMI) na maior parte das espécies. Os ácidos graxos poliinsaturados (AGPI) apresentaram altas concentrações em P. tricornutum e T. gracilis. Dessa 
Tabela 2 - Composição de ácidos graxos de cinco espécies de microalgas na fase estacionária de crescimento ${ }^{1}$.

\begin{tabular}{|c|c|c|c|c|c|}
\hline Ácidos graxos & $\begin{array}{c}\text { Chlorella } \\
\text { minutissima }\end{array}$ & $\begin{array}{c}\text { Dunaliella } \\
\text { tertiolecta }\end{array}$ & Isochrysis galbana & $\begin{array}{l}\text { Nannochloropsis } \\
\text { oculata }\end{array}$ & $\begin{array}{l}\text { Phaeodactylum } \\
\text { tricornutum }\end{array}$ \\
\hline & \multicolumn{5}{|c|}{ Percentagem de ácidos graxos } \\
\hline \multicolumn{6}{|l|}{ Saturados } \\
\hline 08:0 & $4,1 \pm 0,2$ & $8,0 \pm 0,8$ & $1,3 \pm 0,1$ & $1,4 \pm 0,1$ & $0,4 \pm 0,0$ \\
\hline 10:0 & $2,6 \pm 0,1$ & $7,8 \pm 0,4$ & $0,4 \pm 0,1$ & $1 \pm 0,0$ & $0,5 \pm 0,1$ \\
\hline $12: 0$ & $1,5 \pm 0,0$ & $1,0 \pm 0,0$ & - & - & $0,25 \pm 0,1$ \\
\hline $14: 0$ & $4,4 \pm 0,3$ & $4,3 \pm 0,3$ & $14,3 \pm 1,2$ & $1,1 \pm 0,1$ & $15,1 \pm 1,4$ \\
\hline $15: 0$ & $1,0 \pm 0,1$ & $0,7 \pm 0,1$ & $0,9 \pm 0,1$ & - & $0,55 \pm 0,1$ \\
\hline $16: 0$ & $18 \pm 0,7$ & $16,7 \pm 0,8$ & $14,5 \pm 0,5$ & $27,2 \pm 1,1$ & $10 \pm 0,4$ \\
\hline $17: 0$ & - & - & - & $0,7 \pm 0,1$ & $1,3 \pm 0,1$ \\
\hline $18: 0$ & $6,3 \pm 0,3$ & - & $6,1 \pm 0,4$ & $3,1 \pm 0,3$ & $6,9 \pm 0,1$ \\
\hline $20: 0$ & - & - & $1,0 \pm 0,1$ & - & - \\
\hline $21: 0$ & - & - & - & - & - \\
\hline $24: 0$ & - & - & - & - & - \\
\hline Soma & $38,0 \pm 0,2$ & $41,8 \pm 0,2$ & $38,7 \pm 0,4$ & $34,6 \pm 1,7$ & $35,0 \pm 1,2$ \\
\hline \multicolumn{6}{|c|}{ Monoinsaturados } \\
\hline $14: 1$ & $5,5 \pm 0,1$ & $3,1 \pm 0,4$ & $12,7 \pm 0,4$ & $3,9 \pm 0,4$ & $5,1 \pm 0,1$ \\
\hline $16: 1$ & $8,0 \pm 0,2$ & $9,0 \pm 0,4$ & $3,3 \pm 0,2$ & $23,5 \pm 0,9$ & $11,8 \pm 1,0$ \\
\hline $18: 1$ & $12,7 \pm 0,8$ & $9,8 \pm 0,4$ & $12,2 \pm 0,5$ & $7,8 \pm 0,3$ & $2,8 \pm 0,2$ \\
\hline $20: 1$ & - & - & $1,05 \pm 0,1$ & - & - \\
\hline Soma & $26,3 \pm 1,0$ & $22,0 \pm 0,4$ & $29,4 \pm 0,3$ & $35,3 \pm 1,6$ & $19,8 \pm 0,9$ \\
\hline \multicolumn{6}{|l|}{ Poliinsaturados } \\
\hline $16: 2$ & $2,8 \pm 0,3$ & $2,3 \pm 0,1$ & $0,35 \pm 0,1$ & $1,6 \pm 0,1$ & $1,8 \pm 0,1$ \\
\hline $16: 3$ & $6,6 \pm 0,5$ & $1,9 \pm 0,1$ & $0,65 \pm 0,1$ & - & $0,6 \pm 0,1$ \\
\hline $16: 4$ & - & $7,5 \pm 0,2$ & - & - & $8,3 \pm 0,3$ \\
\hline $18: 2$ & $4,7 \pm 0,3$ & $4 \pm 0,7$ & $5,3 \pm 0,4$ & $3,8 \pm 0,1$ & $1,9 \pm 0,0$ \\
\hline $18: 3$ & $17,5 \pm 0,6$ & $14,8 \pm 0,3$ & $4,2 \pm 0,3$ & $1,2 \pm 0,1$ & $1,5 \pm 0,1$ \\
\hline $18: 4$ & - & - & $10,7 \pm 0,6$ & $4,4 \pm 0,3$ & - \\
\hline $20: 4$ & - & $0,7 \pm 0,0$ & - & $2,8 \pm 0,4$ & $0,55 \pm 0,1$ \\
\hline $20: 5$ & $0,8 \pm 0,1$ & $0,4 \pm 0,1$ & - & $12,9 \pm 0,8$ & $24,6 \pm 0,9$ \\
\hline $22: 5$ & $1,1 \pm 0,1$ & $1,1 \pm 0,1$ & - & - & $1,2 \pm 0,1$ \\
\hline $22: 5$ & - & - & $6,4 \pm 0,4$ & - & $1,3 \pm 0,1$ \\
\hline Soma & $33,6 \pm 0,3$ & $32,9 \pm 0,9$ & $27,6 \pm 0,3$ & $26,8 \pm 0,3$ & $41,9 \pm 0,7$ \\
\hline
\end{tabular}

${ }^{1}$ Os resultados referem-se à média de duas repetições \pm desvio padrão.

forma, microalgas podem ser fontes potenciais de AGS, pois plantas terrestres apresentam baixas concentrações desses compostos, exceto espécies oleaginosas. A abundância de AGS e de AGMI entre 14 e 18 carbonos aponta para o uso dessas espécies como matéria-prima para a produção de biodiesel (CHISTI, 2007). Plantas como soja e canola contêm altas concentrações de AGPI, principalmente ácidos linoleico (18:2n-6) e alfa-linoleico (18:3n-3). C. minutissima, $\boldsymbol{D}$. tertiolecta e $\boldsymbol{T}$. gracilis apresentaram altas concentrações de ácido linoleico. Altos teores desse ácido graxo foram encontrados por ZHUKOVA \& AIZDACHER (1995) em espécies dos mesmos gêneros. P. tricornutum apresentou altas concentrações de ácido eicosapentaenoico (20:5, EPA), o que está de acordo com dados da literatura e confirma seu potencial como alimento para animais aquáticos (LOURENCO et al., 2002). N. oculata apresentou baixas concentrações desse AGPI, embora esse gênero apresente altas concentrações. Esse fato pode ser atribuído às baixas taxas de crescimento da espécie e ao excesso de nutrientes no meio de cultivo. Embora as espécies tenham apresentado alguns ácidos graxos em abundância relativamente alta, para que possam ser utilizadas como fonte de lipídeos, é necessária a adoção de condições de cultivo que estimulem a produção desses constituintes pelas microalgas.

Os ácidos aminados mais abundantes foram o glutâmico e o aspártico, ao passo que histidina e metionina foram os menos expressivos (Tabelas 4 e 5), 
Tabela 3 - Composição de ácidos graxos de cinco espécies de microalgas na fase estacionária de crescimento ${ }^{1}$.

\begin{tabular}{|c|c|c|c|c|c|}
\hline Ácidos graxos & $\begin{array}{l}\text { Prorocentrum } \\
\text { minimum }\end{array}$ & Rhodomonas sp. & $\begin{array}{l}\text { Skeletonema } \\
\text { costatum }\end{array}$ & $\begin{array}{l}\text { Synechococcus } \\
\text { subsalsus }\end{array}$ & Tetraselmis gracilis \\
\hline \multicolumn{6}{|c|}{ Percentagem de ácidos graxos } \\
\hline \multicolumn{6}{|l|}{ Saturados } \\
\hline 08:0 & $10,2 \pm 0,4$ & $6,2 \pm 0,4$ & $2,1 \pm 0,2$ & $1,3 \pm 0,1$ & $0,55 \pm 0,1$ \\
\hline $10: 0$ & $1,7 \pm 0,3$ & $7,7 \pm 0,3$ & $3,0 \pm 0,2$ & $0,35 \pm 0,1$ & $1,2 \pm 0,1$ \\
\hline $12: 0$ & - & $2,2 \pm 0,1$ & $0,8 \pm 0,1$ & $0,6 \pm 0,1$ & $0,9 \pm 0,1$ \\
\hline $14: 0$ & $2,5 \pm 0,0$ & $4,2 \pm 0,4$ & $7,2 \pm 0,3$ & $33,8 \pm 1,2$ & $12 \pm 0,7$ \\
\hline $15: 0$ & $1,1 \pm 0,1$ & $0,7 \pm 0,1$ & $1,5 \pm 0,2$ & $1,0 \pm 0,1$ & $0,85 \pm 0,1$ \\
\hline $16: 0$ & $32,1 \pm 1,2$ & $17,8 \pm 0,6$ & $14,7 \pm 0,2$ & $14,8 \pm 0,6$ & $17 \pm 0,3$ \\
\hline $17: 0$ & - & $0,7 \pm 0,1$ & $1,8 \pm 0,2$ & $0,4 \pm 0,1$ & $0,35 \pm 0,1$ \\
\hline 18:0 & $6,1 \pm 0,1$ & $6,1 \pm 0,3$ & $3 \pm 0,0$ & $7,8 \pm 0,3$ & $4,5 \pm 0,3$ \\
\hline $20: 0$ & - & - & - & $0,5 \pm 0,1$ & - \\
\hline $21: 0$ & - & - & - & - & - \\
\hline $24: 0$ & $3,3 \pm 0,2$ & - & - & - & - \\
\hline Soma & $57,2 \pm 2,0$ & $45,7 \pm 0,1$ & $34,4 \pm 0,4$ & $60,7 \pm 2,2$ & $37,3 \pm 1,5$ \\
\hline \multicolumn{6}{|c|}{ Monoinsaturados } \\
\hline $14: 1$ & $6,2 \pm 0,3$ & $7,5 \pm 0,4$ & $19,3 \pm 1,1$ & $5,4 \pm 0,3$ & $3,0 \pm 0,2$ \\
\hline $16: 1$ & $4,8 \pm 0,2$ & $4,6 \pm 0,2$ & $17,6 \pm 0,6$ & $7,7 \pm 0,3$ & $3,7 \pm 0,4$ \\
\hline $18: 1$ & $10,2 \pm 0,6$ & $14,9 \pm 0,6$ & $6,8 \pm 0,6$ & $5,5 \pm 0,2$ & $8,4 \pm 0,8$ \\
\hline $20: 1$ & $1,4 \pm 0,2$ & - & - & $0,3 \pm 0,1$ & $0,3 \pm 0,1$ \\
\hline Soma & $22,8 \pm 1,3$ & $27,1 \pm 1,1$ & $43,8 \pm 1,1$ & $19,0 \pm 0,3$ & $15,6 \pm 0,6$ \\
\hline \multicolumn{6}{|c|}{ Poliinsaturados } \\
\hline $16: 2$ & - & $1,8 \pm 0,1$ & $1,6 \pm 0,1$ & $0,4 \pm 0,1$ & $2,0 \pm 0,1$ \\
\hline $16: 3$ & - & - & $1,3 \pm 0,1$ & - & $0,8 \pm 0,1$ \\
\hline $16: 4$ & - & - & $1,7 \pm 0,1$ & $0,6 \pm 0,1$ & $8,9 \pm 0,1$ \\
\hline $18: 2$ & $3,7 \pm 0,1$ & $2,1 \pm 0,1$ & $2,7 \pm 0,3$ & $3,3 \pm 0,3$ & $2,4 \pm 0,1$ \\
\hline $18: 3$ & $4,0 \pm 0,2$ & $6,0 \pm 0,5$ & $2,5 \pm 0,2$ & $10,2 \pm 0,1$ & $13,9 \pm 0,4$ \\
\hline $18: 4$ & $2,3 \pm 0,3$ & $5,5 \pm 0,3$ & - & $0,6 \pm 0,1$ & $5,1 \pm 0,3$ \\
\hline $20: 4$ & $2,1 \pm 0,2$ & $0,5 \pm 0,1$ & $1,9 \pm 0,1$ & $1,8 \pm 0,1$ & $0,7 \pm 0,1$ \\
\hline $20: 5$ & $2,2 \pm 0,3$ & $6,5 \pm 0,2$ & $1,7 \pm 0,0$ & $0,9 \pm 0,1$ & $7,6 \pm 0,6$ \\
\hline $22: 5$ & $2,7 \pm 0,2$ & $1,7 \pm 0,1$ & $4,3 \pm 0,3$ & - & $0,65 \pm 0,1$ \\
\hline $22: 5$ & $2,0 \pm 0,1$ & $1,3 \pm 0,2$ & $0,8 \pm 0,1$ & - & - \\
\hline Soma & $19,2 \pm 0,8$ & $25,7 \pm 0,1$ & $18,7 \pm 0,1$ & $18,0 \pm 0,4$ & $42,3 \pm 0,4$ \\
\hline
\end{tabular}

${ }^{1}$ Os resultados referem-se à média de duas repetições \pm desvio padrão.

concordando com LOURENÇO et al. (1998). Os padrões de ácidos aminados foram semelhantes entre as espécies, corroborando BECKER (2007), que observa padrões similares para outras espécies de microalgas e fontes proteicas diversas (ovo e soja). Entretanto, a presença de lisina e arginina em concentrações relativamente altas sugere que estas podem ser utilizadas em associação com o farelo de soja no arraçoamento animal, especialmente considerando a piscicultura, a fim de proporcionar melhor equilíbrio de aminoácidos, pois, segundo COLDEBELLA \& RADÜNZ NETO (2002), a alimentação de peixes com farelo de soja requer suplementação desses ácidos aminados, que pode ser obtida de outras fontes vegetais, como as microalgas em questão.

\section{CONCLUSÕES}

O crescimento e a composição química das microalgas variam amplamente. As proteínas são as substâncias mais abundantes, ao passo que carboidratos e lipídeos apresentaram baixas concentrações.

Submetidas às condições de cultivo adotadas, as espécies I. galbana e T. gracilis apresentam o melhor balanço entre produção de biomassa e conteúdos de proteínas, carboidratos e lipídeos, sendo espécies indicadas para testes em maior escala de produção. As condições de cultivo empregadas neste estudo são estimulantes para a produção de ácido alfa-linoleico por T. gracilis e $\boldsymbol{D}$. tertiolecta, indicando o potencial dessas espécies como 
Tabela 4 - Ácidos aminados totais de cinco microalgas marinhas na fase estacionária de crescimento ${ }^{1}$.

\begin{tabular}{|c|c|c|c|c|c|}
\hline Ácidos aminados & $\begin{array}{c}\text { Chlorella } \\
\text { minutissima }\end{array}$ & Dunaliella tertiolecta & Isochrysis galbana & $\begin{array}{c}\text { Nannochloropsis } \\
\text { oculata }\end{array}$ & $\begin{array}{l}\text { Prorocentrum } \\
\text { minimum }\end{array}$ \\
\hline \multicolumn{6}{|c|}{ Percentagem de ácidos aminados } \\
\hline Ácido cisteico & $0,4 \pm 0,1$ & $0,7 \pm 0,2$ & $0,6 \pm 0,0$ & $0,3 \pm 0,0$ & $0,8 \pm 0,1$ \\
\hline Ácido aspártico & $10,8 \pm 1,1$ & $12,1 \pm 0,3$ & $13,0 \pm 0,5$ & $11,7 \pm 0,4$ & $10,4 \pm 0,2$ \\
\hline Treonina & $5,4 \pm 0,3$ & $5,0 \pm 0,2$ & $5,4 \pm 0,4$ & $5,4 \pm 0,1$ & $5,5 \pm 0,3$ \\
\hline Serina & $6,3 \pm 0,6$ & $4,0 \pm 0,3$ & $4,1 \pm 0,3$ & $3,6 \pm 0,0$ & $4,2 \pm 0,4$ \\
\hline Ác. glutâmico & $11,1 \pm 1,2$ & $12,8 \pm 0,4$ & $11,8 \pm 0,2$ & $14,9 \pm 0,5$ & $12,3 \pm 0,2$ \\
\hline Prolina & $6,6 \pm 0,5$ & $4,8 \pm 0,2$ & $4,1 \pm 0,3$ & $5,9 \pm 0,2$ & $4,8 \pm 0,5$ \\
\hline Glicina & $5,2 \pm 0,1$ & $5,6 \pm 0,3$ & $5,2 \pm 0,2$ & $6,2 \pm 0,5$ & $8,0 \pm 0,4$ \\
\hline Alanina & $9,6 \pm 1,0$ & $7,0 \pm 0,2$ & $7,2 \pm 0,3$ & $6,0 \pm 0,2$ & $7,6 \pm 0,2$ \\
\hline Valina & $8,3 \pm 0,7$ & $5,5 \pm 0,5$ & $6,7 \pm 0,2$ & $5,7 \pm 0,6$ & $5,6 \pm 0,4$ \\
\hline Metionina & $2,6 \pm 0,1$ & $2,8 \pm 0,3$ & $2,2 \pm 0,2$ & $2,2 \pm 0,3$ & $2,3 \pm 0,2$ \\
\hline Isoleucina & $5,8 \pm 0,1$ & $4,1 \pm 0,3$ & $5,5 \pm 0,2$ & $4,9 \pm 0,1$ & $4,6 \pm 0,3$ \\
\hline Leucina & $7,9 \pm 0,8$ & $8,7 \pm 0,4$ & $9,5 \pm 0,3$ & $8,4 \pm 0,3$ & $8,2 \pm 0,3$ \\
\hline Tirosina & $4,3 \pm 0,2$ & $3,3 \pm 0,1$ & $3,4 \pm 0,2$ & $3,2 \pm 0,3$ & $3,6 \pm 0,2$ \\
\hline Fenilalanina & $6,3 \pm 0,5$ & $5,4 \pm 0,3$ & $5,8 \pm 0,1$ & $5,3 \pm 0,2$ & $6,1 \pm 0,1$ \\
\hline Histidina & $1,5 \pm 0,3$ & $2,1 \pm 0,3$ & $2,0 \pm 0,2$ & $2,1 \pm 0,3$ & $2,3 \pm 0,4$ \\
\hline Lisina & $5,1 \pm 0,4$ & $5,4 \pm 0,3$ & $5,4 \pm 0,4$ & $6,7 \pm 0,2$ & $5,8 \pm 0,5$ \\
\hline Arginina & $3,6 \pm 0,3$ & $5,4 \pm 0,4$ & $5,6 \pm 0,4$ & $6,5 \pm 0,1$ & $2,3 \pm 0,3$ \\
\hline Total & $100,8 \pm 4,3$ & $94,7 \pm 5,5$ & $97,5 \pm 3,3$ & $99,0 \pm 3,5$ & $94,4 \pm 3,0$ \\
\hline
\end{tabular}

${ }^{1}$ Os resultados representam a percentagem de cada aminoácido em $100 \mathrm{~g}$ de proteínas algáceas, indicando a recuperação real dos ácidos aminados após as análises. Os valores referem-se à média de três repetições \pm desvio padrão $(\mathrm{n}=3)$.

Tabela 5 - Ácidos aminados totais de cinco microalgas marinhas na fase estacionária de crescimento ${ }^{1}$.

\begin{tabular}{|c|c|c|c|c|c|}
\hline Ácidos aminados & $\begin{array}{c}\text { Phaeodactylum } \\
\text { tricornutum }\end{array}$ & Rhodomonas sp. & $\begin{array}{l}\text { Skeletonema } \\
\text { costatum }\end{array}$ & $\begin{array}{c}\text { Synechococcus } \\
\text { subsalsus }\end{array}$ & Tetraselmis gracilis \\
\hline \multicolumn{6}{|c|}{ Percentagem de ácidos aminados } \\
\hline Ác. cisteico & $0,4 \pm 0,0$ & $0,8 \pm 0,2$ & $0,3 \pm 0,0$ & $0,4 \pm 0,1$ & $0,6 \pm 0,1$ \\
\hline Ác. aspártico & $14,9 \pm 0,7$ & $13,4 \pm 0,2$ & $13,5 \pm 0,1$ & $13,0 \pm 0,6$ & $11,4 \pm 0,4$ \\
\hline Treonina & $5,0 \pm 0,3$ & $4,7 \pm 0,0$ & $5,6 \pm 0,1$ & $5,5 \pm 0,3$ & $5,7 \pm 0,2$ \\
\hline Serina & $4,5 \pm 0,2$ & $3,4 \pm 0,1$ & $4,2 \pm 0,1$ & $3,4 \pm 0,3$ & $3,8 \pm 0,3$ \\
\hline Ác. glutâmico & $3,0 \pm 0,8$ & $11,5 \pm 0,4$ & $13,8 \pm 0,3$ & $16,1 \pm 0,2$ & $13,9 \pm 0,8$ \\
\hline Prolina & $3,7 \pm 0,3$ & $3,3 \pm 0,2$ & $3,9 \pm 0,4$ & $3,7 \pm 0,3$ & $4,8 \pm 0,3$ \\
\hline Glicina & $5,2 \pm 0,2$ & $6,7 \pm 0,5$ & $6,0 \pm 0,3$ & $5,6 \pm 0,2$ & $6,4 \pm 0,4$ \\
\hline Alanita & $6,8 \pm 0,4$ & $6,9 \pm 0,2$ & $6,6 \pm 0,2$ & $7,9 \pm 0,4$ & $7,0 \pm 0,4$ \\
\hline Valina & $6,0 \pm 0,3$ & $5,7 \pm 0,3$ & $5,3 \pm 0,3$ & $5,9 \pm 0,2$ & $6,5 \pm 0,4$ \\
\hline Metionina & $2,5 \pm 0,3$ & $2,4 \pm 0,4$ & $2,9 \pm 0,2$ & $3,5 \pm 0,2$ & $1,7 \pm 0,3$ \\
\hline Isoleucina & $5,3 \pm 0,2$ & $4,8 \pm 0,1$ & $5,9 \pm 0,3$ & $5,5 \pm 0,2$ & $4,5 \pm 0,4$ \\
\hline Leucina & $7,8 \pm 0,4$ & $7,5 \pm 0,3$ & $8,2 \pm 0,2$ & $9,7 \pm 0,3$ & $8,9 \pm 0,6$ \\
\hline Tirosina & $3,0 \pm 0,1$ & $4,9 \pm 0,3$ & $3,5 \pm 0,2$ & $3,4 \pm 0,3$ & $2,9 \pm 0,2$ \\
\hline Fenilalanina & $5,6 \pm 0,2$ & $5,3 \pm 0,4$ & $6,3 \pm 0,1$ & $5,1 \pm 0,4$ & $6,1 \pm 0,4$ \\
\hline Histidina & $1,7 \pm 0,3$ & $1,6 \pm 0,2$ & $1,7 \pm 0,3$ & $1,9 \pm 0,2$ & $2,5 \pm 0,3$ \\
\hline Lisina & $5,5 \pm 0,3$ & $5,2 \pm 0,0$ & $4,4 \pm 0,4$ & $4,8 \pm 0,2$ & $6,4 \pm 0,3$ \\
\hline Arginina & $5,8 \pm 0,1$ & $4,5 \pm 0,3$ & $4,4 \pm 0,2$ & $6,0 \pm 0,3$ & $6,7 \pm 0,7$ \\
\hline Total & $96,7 \pm 3,6$ & $92,6 \pm 3,2$ & $96,5 \pm 3,5$ & $101,4 \pm 3,9$ & $99,8 \pm 3,4$ \\
\hline
\end{tabular}

\footnotetext{
${ }^{1}$ Os resultados representam a percentagem de cada aminoácido em $100 \mathrm{~g}$ de proteínas algáceas, indicando a recuperação real dos ácidos aminados após as análises. Os valores referem-se à média de três repetições \pm desvio padrão $(\mathrm{n}=3)$.
}

Ciência Rural, v.40, n.2, fev, 2010. 
fontes alternativas dessas substâncias. Além disso, as condições de cultivo adotadas também são estimulantes para a produção de carotenoides por $\boldsymbol{D}$. tertiolecta e EPA por $\boldsymbol{P}$. tricornutum, determinando a importância dessas espécies para a produção desses ácidos graxos. Desse modo, submetidas à metodologia adotada neste trabalho, as espécies testadas apresentam composições de ácidos aminados semelhantes em concentrações adequadas para uso como alimento na aquicultura.

\section{REFERÊNCIAS}

BECKER, E.W. Micro-algae as a source of protein. Biotechnology Advances, n.25, p.207-210, 2007. Disponível em: <http://www.sciencedirect.com/ science?_ob=ArticleURL\&_udi=B6T4X-4MDGP 8F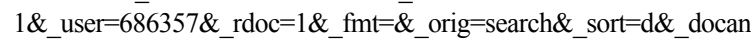 $\mathrm{chor}=\&$ view $=\bar{c}_{\text {\& }}$ acct $=\mathrm{C} 000037518 \&$ \& version $=1 \&$ _ u r 1 Ve r s i o $n=0 \& \_$u s e r i d $=686357 \&$ m $\mathrm{d} 5=\mathrm{c} 85369 \mathrm{df} 880 \mathrm{a} 1190 \mathrm{fb} 8 \mathrm{dcd} 89670 \mathrm{a} 1 \mathrm{~b} 2 \mathrm{a}>$. Acesso em: 15 jan. 2008. doi: 10.1016/j.biotechadv.2006.11.002.

BROWN, M.R. et al. Nutritional properties of microalgae for mariculture. Aquaculture, v.151, p.315-331, 1997. Disponível em: <http:// www.sciencedirect.com/science?_ob=ArticleURL\&_udi=B6T4D3RH6WPV-X\&_user $=686357 \&$ _rdoc $=1 \&$ fmt $=$ \&_orig $=$ search

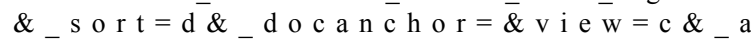
$\mathrm{c} c \mathrm{t}=\mathrm{C} 000037518 \&$ \& e r s i o $=1 \&$ \& ur 1 Versi

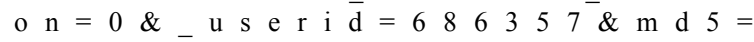
849414074b0e5b998f281b13fe3e6360>. Acesso em: 20 jul. 2008. doi: 10.1016/S0044-8486(96)01501-3.

BRZEZINSKI, M.A. The Si-C-N ratio of marine diatoms interspecific variability and the effect of some environmental variables. Journal of Phycology, v.21, n.2, p.347-357, 1985. Disponível em: <http://www3.interscience.wiley.com/journal/ 119508756/issue $>$. Acesso em: 20 jun. 2008. doi: 10.1111/ j.0022-3646.1985.00341.x.

COLDEBELLA, I.J.; RADNÜNZ NETO, J. Farelo de soja na alimentação de alevinos de jundiá (Rhamdia quelen). Ciência Rural, v.32, n.3, p.499-503, 2002. Disponível em: <http:// www.scielo.br/scielo.php? script $=$ sci_arttext\&pid $=$ S 0103 $84782002000300021 \& \operatorname{lng}=\mathrm{en} \& \mathrm{nrm}=\mathrm{isso}>$. Acesso em: 14 set. 2008. doi: 10.1590/S0103-84782002000300021.

CHISTI, Y. Biodiesel from microalgae. Biotechnology Advances, v.25, n.3, p.294-306, 2007. Disponível em: <http:// www.sciencedirect.com/science?_ob=ArticleURL\&_udi=B6T4X4 N20704-1\&_user $=686357 \&$ rdoc $=1 \&$ fmt $=$ \&_orig $=$ search $\&$ _sort $=$ d\&_docanchor $=\&$ view $=$ c \&_a c c t $=$ C 0000 $37518 \&$ \& version $=1 \&$ _ur 1 Version $=0$ \&_userid $=6$ $86357 \& \mathrm{md} 5=\mathrm{c} 72 \mathrm{dcf} 414 \mathrm{f} 40 \mathrm{fl}-\overline{\mathrm{a}} 80 \mathrm{cb} 535 \mathrm{f} 012753 \mathrm{e} 1 \mathrm{e}>$. Acesso em: 15 jan. 2008. doi: 10.1016/j.biotechadv.2007.02.001.

DE PAWN, N. et al. Mass culture of microalgae in aquaculture systems progress and constraints. Hydrobiologia, v.116/117, p.121-134, 1984. Disponível em: <http://www.springerlink.com.w10033.dotlib.com.br/

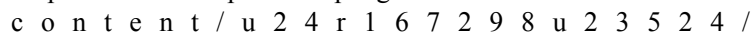
?p $=$ db5ddcd0686b4e05a619eef6cflad1d3\&pi=21 >. Acesso em: 13 mar. 2008. doi: 10.1007/BF00027650.
DUBOIS, M. et al. Colorimetric method for determination of sugars and related substances. Analytical Chemistry, v.28, p.350-356, 1956. Disponível em: <http://pubs.acs.org/doi/abs/ $10.1021 /$ a c 60111 a 017 ? prevSearch=\%255 B a th or\%253A\%2BDubois\%255D\&searchHistoryKey $=>$. Acesso em: 02 fev. 2008. doi: 10.1021/ac60111a017.

FOLCH, J. et al. A simple method for the isolation and purification of total lipid from animal tissue. Journal of Biological Chemistry, v.226, p.497-509, 1957. Disponível em: <http://intl.jbc.org/search?author $1=$ Folch \&fulltext $=\& p u$ bdate_year $=\&$ volume $=\&$ firstpage $=\&$ submit $=$ yes $>$.

HARTMAN, L.; LAGO, B.C.A. Rapid preparation of fatty acid methyl esters from lipids. Laboratory Practice, v.22, p.475-477, 1973.

HILLEBRAND, H. et al. Biovolume calculation for pelagic and benthic microalgae. Journal of Phycology, v.35, n.2, p.403424, 1999. Disponível em: <http://www3.interscience.wiley.com/ journal/119064399/issue $>$. Acesso em: 18 mai. 2008. doi: 10.1046/j.1529-8817.1999.3520403.x

JEFFREY, S.W.; HUMPHREY, G.F. New spectrophotometric equations for determining chlorophylls $\mathrm{a}, \mathrm{b}, \mathrm{c} 1$ and $\mathrm{c} 2$ in higher plants, algae and natural phytoplankton. Biochemistry and Physiology Pflanzen, v.167, p.191-194, 1975.

LAVÍN, P.L.; LOURENÇO, S.O. An evaluation of the accumulation of intracellular inorganic nitrogen pools by marine microalgae in cultures. Brazilian Journal of Oceanography, v.53, n1/2, p.55-67, 2005. Disponível em: <http:// www.scielo.br/scielo.php? script $=$ sci_abstract\&pid $=$ S 1679 $87592005000100006 \& \operatorname{lng}=\mathrm{en} \& \mathrm{nrm}=\overline{\mathrm{is}} \& \mathrm{t} \operatorname{lng}=\mathrm{pt}>$. Acesso em: 02 mar. 2008. doi: 10.1590/S1679-87592005000100006.

LI et al. Biofuels from microalgae. Biotechnology Progress, v.24, n.4, p.815-820, 2008. Disponível em: <http://apps.isiknowledge.com/ full_record.do?product $=U A \&$ search_mode $=$ GeneralSearch \& $\mathrm{q} \mathrm{i}^{-} \mathrm{d}=1 \& \mathrm{~S}$ I D $=3$ B $4 \mathrm{P} \mathrm{P}^{-} \mathrm{N}$ n $\mathrm{G}$ f e p $3 \mathrm{~B}$ $\mathrm{dLN} 4 \mathrm{~m} \&$ page $=4 \& \mathrm{doc}=31 \&$ colname $=\mathrm{WOS}>$. Acesso em: $02 \mathrm{mai}$ 2008. doi: $10.1021 / \mathrm{bp} .070371 \mathrm{k}$.

LORENZEN, C.J. Determination of chlorophyll and phaeophytin: spectrophotometric equations. Limnology and Oceanography, v.12, p.343-346, 1967.

LOURENÇO, S.O. Cultivo de microalgas marinhas: princípios e aplicações. São Carlos: RiMa, 2006. 606 p.

LOURENÇO, S.O. et al. Distribution of intracellular nitrogen in marine microalgae: calculation of new nitrogen-to-protein conversion factors. European Journal of Phycology, v.39, n.1, p.17-32, 2004. Disponível em: <http://www.informaworld.com/smpp/ section? content $=\mathrm{a} 713821622 \&$ fulltext $=713240928>$. Acesso em: 05 mai 2008. doi: 10.1080/0967026032000157156.

LOURENÇO, S.O. et al. Effects of different nitrogen sources on growth and biochemical profile of ten marine microalgae under batch cultures: an evaluation for aquaculture. Phycologia, v.41, n.2, p.158-168, 2002.

LOURENÇO, S.O. et al. Distribution of intracellular nitrogen in marine microalgae: Basis for the calculation of specific nitrogen-to-protein conversion factors. Journal of Phycology, v.34, n.5, p.798-811, 1998. Disponível em: <http:// 
www3.interscience.wiley.com/journal/119110659/issue>. doi: 10.1046/j.1529-8817.1998.340798.x

LOWRY, O.H. et al. Protein measurement with the folin phenol reagent. Journal of Biological Chemistry, v.193, p.265-275, 1951. Disponível em: <http://intl.jbc.org/ search ? a u thor $1=$ L ow y $\&$ fu 11 text $=\&$ pubdate _year $=1951 \&$ volume $=\&$ firstpage $=\&$ submit $=$ yes $>$.

MYKLESTAD, S.; HAUG, A. Production of carboydrates by the marine diatom Chaetoceros affinis var. Willei (Gran) Hustedt. I. Effect of the concentration of nutrients in the culture medium. Journal of Experimental Marine Biology and Ecology, v.9, p.125-136, 1972. Disponível em: <http://www.sciencedirect.com/ science? ob=ArticleURL\& udi=B 6 T 8 F-489S4VX$1 \&$ user $=686357 \&$ coverDate $=12 \% 2$ F $31 \% 2$ F $1972 \&$ _alid $=1023143944 \&$ rdoc $=18 \&$ fmt $=$ high\&_orig $=$ search \& _cdi $=5085 \&$ \& sort $=$ r\&_docanchor $=\&$ view $=$ c \&_ct $=2$ 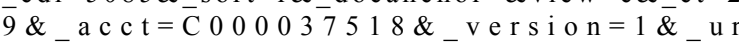 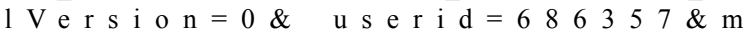 d5 $=$ cca2004ad71 abc81784faa1f734be1f3 $>$. Acesso em: 12 set. 2008. doi: 10.1016/0022-0981(72)90041-X.

PULZ, O.; GROSS, W. Valuable products from biotechnology microalgae. Applied Microbiology Biotechnology, v.65, n.6, p.635-648, 2004. Disponível em: <http:// www.springerlink.com/content/gbbkaptetj1n76h3/fulltext.html>. Acesso em: 22 mai. 2008. doi: 10.1007/s002530100702.

RENAULD, S.M. et al. The gross chemical composition and fatty acid composition of 18 species of tropical Australian microalgae for possible use in mariculture. Aquaculture, v.170, n.2, p.147-159, 1999. Disponível em: <http://www.sciencedirect.com/ science? ob=ArticleURL\& udi=B6T4D-3 VFF4YX$5 \&$ _user $=686357 \&$ coverDate $=01 \% 2 \mathrm{~F} 15 \% 2 \mathrm{~F} 1999 \&$ rdoc $=5 \&$

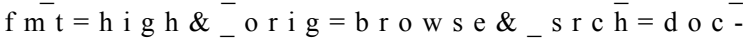
info(\%23 toc \% $234972 \% 231999 \% 23998299997 \% 2$ $344910 \% 23$ F L A \%23 display \%23Volume)\& cdi=4 $972 \&$ s ort $=$ d\& docanchor $\&$ \& c t $=8 \&$ a c
$\mathrm{ct}=\mathrm{C} 000037518 \&$ _ version $=1 \&$ \& ur 1 Versi

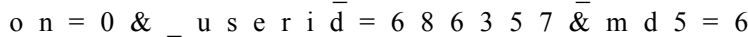
036d856ff8908 $\mathrm{cf} 63 \mathrm{f} 444149 \mathrm{c} 77 \mathrm{ff} 95>$. Acesso em: 25 jul. 2008. doi: $10.1016 / \mathrm{S} 0044-8486(98) 00399-8$.

SKJANES, K. et al. BioCO $_{2}-\mathrm{A}$ multidisciplinary, biological approach using solar energy to capture $\mathrm{CO}_{2}$ while producing $\mathrm{H}_{2}$ and high value products. Biomolecular Engineering, v.24, n.4, p.405-413, 2007. Disponível em: <http://www.sciencedirect.com/ science?_ob=ArticleURL\&_udi=B6VRM-4P12J83$1 \&$ \& s e r $=686357 \&$ c o ver D a t e $=10 \% 2$ F 31

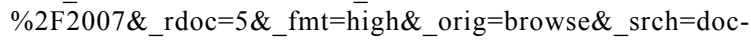
info(\%23 toc\%236238\%232007\%23999759995\%23 $668634 \% 23$ FLA \%23 display\%23 Volume)\&_cdi=6238\& sort $=$ d\& docanchor $=\& \quad$ ct $=7 \& \quad$ acct $=$ C 0000 $37518 \&$ \& version $=1 \&$ \& ur l Vers i o $n=0 \&$ u serid $=68635 \overline{7} \& \mathrm{md} 5=\mathrm{e} 6 \mathrm{f} 636 \mathrm{c} 164 \overline{7} \mathrm{ae} 018 \mathrm{ed} 4043 \mathrm{da} 4 \mathrm{c} 550 \mathrm{a} 4 \overline{\mathrm{a}}>$. Acesso em: 02 set. 2008. doi: 10.1016/j.bioeng.2007.06.002.

STRICKLAND, J.D.H.; PARSONS, T.R. A pratical handbook of seawater analysis. Bulletin of Fisheries Research Board of Canada, v.167, p.1-311, 1968.

TURPIN, D.H. Effects of inorganic $\mathrm{N}$ availability on algal photosynthesis and carbon metabolism. Journal of Phycology, v.27, n.1, p.14-20, 1991. Disponível em: <http:// www3.interscience.wiley.com/journal/119345902/issue >. Acesso em: 18 mai. 2008. doi: 10.1111/j.0022-3646.1991.00014.x.

WALNE, P.R. Experiments in the large scale culture of the larvae of Ostrea edulis. Fishery Investigations, v.25, n.4, p.1-53, 1966.

ZAR, J.H. Biostatistical analysis. 3.ed. Upper Saddle River: Prentice Hall, 1996. 920p.

ZHUKOVA, N.V.; AIZDACHER, N.A. Fatty acid composition of 15 marine microalgae. Phytochemistry, v.39, n.2, p.351356, 1995. 